\title{
On almost polynomial structures from classical linear connections
}

\begin{abstract}
Let $\mathcal{M} f_{m}$ be the category of $m$-dimensional manifolds and local diffeomorphisms and let $T$ be the tangent functor on $\mathcal{M} f_{m}$. Let $\mathcal{V}$ be the category of real vector spaces and linear maps and let $\mathcal{V}_{m}$ be the category of $m$-dimensional real vector spaces and linear isomorphisms. Let $w$ be a polynomial in one variable with real coefficients. We describe all regular covariant functors $F: \mathcal{V}_{m} \rightarrow \mathcal{V}$ admitting $\mathcal{M} f_{m}$-natural operators $\tilde{P}$ transforming classical linear connections $\nabla$ on $m$-dimensional manifolds $M$ into almost polynomial $w$-structures $\tilde{P}(\nabla)$ on $F(T) M=\bigcup_{x \in M} F\left(T_{x} M\right)$.
\end{abstract}

1. Introduction. All manifolds considered in the paper are assumed to be Hausdorff, finite dimensional, second countable, without boundaries and smooth (i.e. of class $C^{\infty}$ ). Maps between manifolds are assumed to be of class $C^{\infty}$.

The category of $m$-dimensional manifolds and local diffeomorphisms is denoted by $\mathcal{M} f_{m}$. The category of vector bundles and vector bundle homomorphisms between them is denoted by $\mathcal{V B}$. The category of $m$-dimensional real vector spaces and linear isomorphisms is denoted by $\mathcal{V}_{m}$. The category of finite dimensional real vector spaces and linear maps is denoted by $\mathcal{V}$.

Let $w$ be a polynomial in one variable. A tensor field $P$ of type $(1,1)$ on a manifold $N$ is called an almost polynomial $w$-structure on $N$ if $w(P)=0$ (i.e. $w\left(P_{\mid x}\right)=0$ for any $\left.x \in N\right)$.

2010 Mathematics Subject Classification. 58A20, 58A32.

Key words and phrases. Classical linear connection, almost polynomial structure, Weil bundle, natural operator. 
In the present paper we solve the following problem.

Problem 1. Let $w$ be a polynomial in one variable with real coefficients. We characterize all covariant regular functors $F: \mathcal{V}_{m} \rightarrow \mathcal{V}$ admitting $\mathcal{M} f_{m}$ natural operators $\tilde{P}$ transforming classical linear connections $\nabla$ on $m$-manifolds $M$ into almost polynomial w-structures $\tilde{P}(\nabla)$ on $F(T) M=\bigcup_{x \in M} F\left(T_{x} M\right)$, where $T: \mathcal{M} f_{m} \rightarrow \mathcal{V B}$ denotes the tangent functor on the category $\mathcal{M} f_{m}$.

If $w(t)=t^{2}+1$, then we reobtain the result from [5] on the characterization of covariant regular functors $F: \mathcal{V}_{m} \rightarrow \mathcal{V}$ admitting $\mathcal{M} f_{m}$-natural operators $\tilde{J}$ transforming classical linear connections $\nabla$ on $m$-manifolds $M$ into almost complex structures $\tilde{J}(\nabla)$ on $F(T) M$.

If $w(t)=t^{2}-1$, then we characterize covariant regular functors $F: \mathcal{V}_{m} \rightarrow$ $\mathcal{V}$ admitting $\mathcal{M} f_{m}$-natural operators $\tilde{J}$ transforming classical linear connections $\nabla$ on $m$-manifolds $M$ into almost para-complex structures $\tilde{J}(\nabla)$ on $F(T) M$.

2. Basic definitions. The concept of natural bundles and natural operators can be found in the fundamental monograph [3].

Let $F: \mathcal{V}_{m} \rightarrow \mathcal{V}$ be a covariant regular functor. The regularity of the functor $F$ means that $F$ transforms smoothly parametrized families of isomorphisms into smoothly parametrized families of linear maps. Let $T: \mathcal{M} f_{m} \rightarrow$ $\mathcal{V B}$ be the tangent functor sending any $m$-dimensional manifold $M$ into the tangent bundle $T M$ of $M$ and any $\mathcal{M} f_{m}$-map $\varphi: M_{1} \rightarrow M_{2}$ into the tangent map $T \varphi: T M_{1} \rightarrow T M_{2}$. Applying $F$ to fibers $T_{x} M$ of $T M$, one can define a natural vector bundle $F(T)$ of order 1 over $m$-manifolds by

$$
F(T) M=\bigcup_{x \in M} F\left(T_{x} M\right) \text { and } F(T) \varphi=\bigcup_{x \in M} F\left(T_{x} \varphi\right): F(T) M_{1} \rightarrow F(T) M_{2}
$$

for any $m$-manifold $M$ and any $\mathcal{M} f_{m}$-map $\varphi: M_{1} \rightarrow M_{2}$ between $m$ manifolds $M_{1}$ and $M_{2}$. In particular, if $F$ is the identity functor, then $F(T)=T$.

A classical linear connection on an $m$-manifold $M$ is an $\mathbb{R}$-bilinear map $\nabla: \mathfrak{X}(M) \times \mathfrak{X}(M) \rightarrow \mathfrak{X}(M)$ such that:

(1) $\nabla_{f_{1} X_{1}+f_{2} X_{2}} Y=f_{1} \nabla_{X_{1}} Y+f_{2} \nabla_{X_{2}} Y$

(2) $\nabla_{X}\left(Y_{1}+Y_{2}\right)=\nabla_{X} Y_{1}+\nabla_{X} Y_{2}$

(3) $\nabla_{X}(f Y)=X f \cdot Y+f \cdot \nabla_{X} Y$

where $X, X_{1}, X_{2}, Y, Y_{1}, Y_{2} \in \mathfrak{X}(M)$ are any vector fields on $M$ and $f, f_{1}, f_{2}: M \rightarrow \mathbb{R}$ are any smooth functions on $M$. Equivalently, a classical linear connection on $M$ is a right invariant decomposition $T L M=H^{\nabla} \oplus$ $V L M$ of the tangent bundle $T L M$ of $L M$, where $L M$ is the principal bundle with the structural group $G L(m)$ of linear frames over $M$ and $V L M$ is the vertical bundle of $L M$, see [2]. 
Let $w(t)=t^{m}+a_{m-1} t^{m-1}+\cdots+a_{1} t+a_{0}$ be the polynomial in one variable with real coefficients $a_{m-1}, \ldots, a_{0}$.

A polynomial $w$-structure on a real vector space $W$ is a linear endomorphism $P: W \rightarrow W$ such that $w(P)=P^{m}+a_{m-1} P^{m-1}+\cdots+a_{1} P+a_{0} I=0$, where $P^{k}$ denotes the composition $\underbrace{P \circ \cdots \circ P}_{k \text {-times }}$ and $I$ denotes the identity map on $W$.

An almost polynomial $w$-structure on manifold $N$ is a tensor field $\tilde{P}: T N \rightarrow T N$ on $N$ of type $(1,1)$ (affinor) such that $P_{x}: T_{x} N \rightarrow T_{x} N$ is a polynomial $w$-structure on $T_{x} N$ for any $x \in N$. In other words, an almost polynomial $w$-structure is a tensor field $P$ of type $(1,1)$ on manifold $N$ satisfying a polynomial equation $P^{m}+a_{m-1} P^{m-1}+\cdots+a_{1} P+a_{0} I=0$, where $a_{m-1}, \ldots, a_{0}$ are real numbers, at every point of $N$.

The general concept of natural operators can be found in the fundamental monograph [3]. In particular, we have the following definition.

Definition 1. Let $F: \mathcal{V}_{m} \rightarrow \mathcal{V}$ be a covariant regular functor. An $\mathcal{M} f_{m^{-}}$ natural operator transforming classical linear connections $\nabla$ on $m$-manifolds $M$ into almost polynomial $w$-structures $\tilde{P}(\nabla): T F(T) M \rightarrow T F(T) M$ on $F(T) M$ is an $\mathcal{M} f_{m}$-invariant family $\tilde{P}: Q \rightsquigarrow(A w S) F(T)$ of operators

$$
\tilde{P}: Q(M) \rightarrow(A w S)(F(T) M)
$$

for $m$-manifolds $M$, where $Q(M)$ is the set of classical linear connections on $M$ and $(A w S)(F(T) M)$ is the set of almost polynomial $w$-structures on $F(T) M$. The invariance of $\tilde{P}$ means that if $\nabla_{1} \in Q\left(M_{1}\right)$ and $\nabla_{2} \in$ $Q\left(M_{2}\right)$ are $\varphi$-related by an embedding $\varphi: M_{1} \rightarrow M_{2}$ (i.e. if $\varphi$ is $\left(\nabla, \nabla_{1}\right)$ affine embedding), then $\tilde{P}\left(\nabla_{1}\right)$ and $\tilde{P}\left(\nabla_{2}\right)$ are $F(T) \varphi$-related (i.e. $T F(T) \varphi \circ$ $\left.\tilde{P}\left(\nabla_{1}\right)=\tilde{P}\left(\nabla_{2}\right) \circ T F(T) \varphi\right)$.

Let $F: \mathcal{V}_{m} \rightarrow \mathcal{V}$ be as above. A $\mathcal{V}_{m}$-canonical polynomial $w$-structure on $V \oplus F V$ is a $\mathcal{V}_{m}$-invariant system $P$ of polynomial $w$-structures

$$
P: V \oplus F V \rightarrow V \oplus F V
$$

on vector spaces $V \oplus F V$ for $m$-dimensional real vector spaces $V$. The invariance of $P$ means that $(\varphi \oplus F \varphi) \circ P=P \circ(\varphi \oplus F \varphi)$ for any linear isomorphism $\varphi: V_{1} \rightarrow V_{2}$ between $m$-dimensional vector spaces.

3. The main result. The main result of the present note is the following theorem.

Theorem 1. Let $F: \mathcal{V}_{m} \rightarrow \mathcal{V}$ be a covariant regular functor and $w$ be a polynomial in one variable with real coefficients. The following conditions are equivalent:

(i) There exists an $\mathcal{M} f_{m}$-natural operator $\tilde{P}: Q \rightsquigarrow(A w S) F(T)$.

(ii) There exists a $\mathcal{V}_{m}$-canonical polynomial w-structure $P$ on $V \oplus F V$. 
Proof. $(i) \Rightarrow(i i)$. Let $\tilde{P}: Q \rightsquigarrow(A w S) F(T)$ be an $\mathcal{M} f_{m}$-natural operator in question. Let $V$ be an $m$-dimensional vector space from the category $\mathcal{V}_{m}$ and let $\nabla^{V}$ be the $\mathcal{V}_{m}$-canonical torsion free flat classical linear connection on $V$. Then the almost polynomial $w$-structure $\tilde{P}\left(\nabla^{V}\right): T F(T) V \rightarrow$ $T F(T) V$ on $F(T) V$ restricts to the polynomial $w$-structure

$$
P:=\tilde{P}\left(\nabla^{V}\right)_{0_{0_{V}}}: T_{0_{0_{V}}} F(T) V \rightarrow T_{0_{0_{V}}} F(T) V
$$

on the tangent space $T_{0_{0_{V}}} F(T) V$ of $F(T)(V)$ at $0_{0_{V}} \in F(T) V$, where $0_{V}$ is the zero in $V$ and $0_{0_{V}}$ is the zero in $F(T)_{0_{V}} V$. Since $T V=V \oplus V$, we have $F(T) V=V \oplus F V$. Therefore $T_{0_{0_{V}}} F(T) V=V \oplus F V$ modulo above identifications. So,

$$
P: V \oplus F V \rightarrow V \oplus F V
$$

is the polynomial $w$-structure on $V \oplus F V$ for any $\mathcal{V}_{m}$-object $V$. Because of the canonical character of the construction of $P$, the structure $P$ is $\mathcal{V}_{m^{-}}$ canonical.

$($ ii $) \Rightarrow(i)$. Suppose $P: V \oplus F V \rightarrow V \oplus F V$ is a $\mathcal{V}_{m}$-canonical polynomial $w$-structure. Let $\nabla \in Q(M)$ be a classical linear connection on an $m$-manifold $M$. Let $v \in F(T)_{x} M, x \in M$. Since $F(T)$ is of order $1, F(T) M=L M\left[F(T)_{0} \mathbb{R}^{m}\right]$ (the associated space). Then $\nabla$-decomposition $T L M=H^{\nabla} \oplus V L M$ induces (in obvious way) $\nabla$-decomposition $T F(T) M=$ $\tilde{H}^{\nabla} \oplus V F(T) M$. Then we have the identification

$$
T_{v} F(T) M=\tilde{H}_{v}^{\nabla} \oplus V_{v} F(T) M \cong T_{x} M \oplus F(T)_{x} M=T_{x} M \oplus F\left(T_{x} M\right)
$$

canonically depending on $\nabla$, where the equality is the connection decomposition, the identification $\cong$ is the usual one (namely, $\tilde{H}_{v}^{\nabla}=T_{x} M$ modulo the tangent of the projection of $F(T) M$ and $V_{v} F(T) M=T_{v}\left(F(T)_{x} M\right)=$ $F(T)_{x} M$ modulo the standard identification) and the second equality is by the definition of $F(T) M$. We define $\tilde{P}(\nabla)_{\mid v}: T_{v} F(T) M \rightarrow T_{v} F(T) M$ by

$$
\tilde{P}(\nabla)_{\mid v}:=P: T_{x} M \oplus F\left(T_{x} M\right) \rightarrow T_{x} M \oplus F\left(T_{x} M\right)
$$

modulo the above identification $T_{v} F(T) M \cong T_{x} M \oplus F\left(T_{x} M\right)$. Then $\tilde{P}(\nabla): T F(T) M \rightarrow T F(T) M$ is an almost polynomial $w$-structure on $F(T) M$. By the canonical character of $\tilde{P}(\nabla)$, the resulting family $\tilde{P}: Q \rightsquigarrow(A w S) F(T)$ is an $\mathcal{M} f_{m}$-natural operator.

4. An application to para-complex structures. Let $w(t)=t^{2}-1$. Let $J$ be a polynomial $w$-structure on a vector space $W$. Then $W=W_{+} \oplus W_{-}$, where $W_{ \pm}=\{v \in W: J(v)= \pm v\}$. If additionally $\operatorname{dim}\left(W_{+}\right)=\operatorname{dim}\left(W_{-}\right)$, then $J$ is called a para-complex structure on $W$, see [6].

An almost para-complex structure on a manifold $N$ is an affinor $J: T N \rightarrow$ $T N$ on $N$ such that $J_{x}: T_{x} N \rightarrow T_{x} N$ is a para-complex structure on $T_{x} N$ for any $x \in N$. In other words, an almost para-complex structure is a smooth 
$(1,1)$-tensor field on the manifold $N$ of even dimension $m$, if the following conditions are satisfied:

(1) $J^{2}=i d_{T N}$

(2) for each point $x \in N$, the eigenspaces $T_{x}^{+} N$ and $T_{x}^{-} N$ of $J_{x}$ (the value of $J$ at $x$ ) are both $\frac{m}{2}$-dimensional subspaces of the tangent space $T_{x} N$ at $x,[1],[7]$.

Corollary 1. Let $F: \mathcal{V}_{m} \rightarrow \mathcal{V}$ be a regular covariant functor. The following conditions are equivalent:

(a) There is an $\mathcal{M} f_{m}$-natural operator $\tilde{J}: Q \rightsquigarrow(A P C) F(T)$ transforming classical linear connections $\nabla$ on $m$-manifolds $M$ into almost para-complex structures $\tilde{J}(\nabla)$ on $F(T) M$.

(b) There exists a $\mathcal{V}_{m}$-canonical para-complex structure $J$ on $V \oplus F V$.

Proof. This is a simple consequence of Theorem 1.

Lemma 1. Let $p$ be a positive integer. Let $F: \mathcal{V}_{m} \rightarrow \mathcal{V}$ be a covariant regular functor given by $F V=V \times \cdots \times V((p-1)$ times of $V)$ and $F \varphi=$ $\varphi \times \cdots \times \varphi((p-1)$ times of $\varphi)$. If $p$ is even, there is a $\mathcal{V}_{m}$-canonical para-complex structure on $V \oplus F V$.

Proof. If $p$ is even, we have the $\mathcal{V}_{m}$-canonical para-complex structure on $V \times \cdots \times V(p$ times of $V)$. Namely, we have the $\frac{p}{2}$ copies of the canonical para-complex structure on $V \times V$ given by $(v, w) \rightarrow(v,-w)$.

A Weil algebra $A$ is a finite dimensional, commutative, associative and unital algebra of the form $A=\mathbb{R} \times N$, where $N$ is the ideal of all nilpotent elements of $A$.

Lemma 2 (Lemma 5.1 in [4]). Let $A$ be a p-dimensional Weil algebra and let $T^{A}$ be the corresponding Weil functor. For any classical linear connection $\nabla$ on an $m$-manifold $M$, we have the base-preserving fibred diffeomorphism $I_{\nabla}^{A}: T^{A} M \rightarrow T M \otimes \mathbb{R}^{p-1}$ canonically depending on $\nabla$.

We see that $T M \otimes \mathbb{R}^{p-1}=T M \times_{M} \cdots \times_{M} T M((p-1)$ times of $T M)=$ $F(T) M$, where $F: \mathcal{V}_{m} \rightarrow \mathcal{V}, F V=V \times \cdots \times V((p-1)$ times of $V)$, $F \varphi=\varphi \times \cdots \times \varphi((p-1)$ times of $\varphi)$. So, from Corollary 1, Lemma 1 and Lemma 2 we obtain

Proposition 1. Let $A$ be a Weil algebra. If $A$ is even dimensional, there exists an $\mathcal{M} f_{m}$-natural operator $\tilde{J}: Q \rightsquigarrow(A P C) T^{A}$ sending classical linear connections $\nabla$ on m-manifolds $M$ into almost para-complex structures $\tilde{J}(\nabla)$ on $T^{A} M$.

\section{REFERENCES}

[1] Kaneyuki, S., Kozai, M., Paracomplex structures and affine symmetric spaces, Tokyo J. Math. 8 (1) (1985), 81-98. 
[2] Kobayashi, S., Nomizu, K., Foundations of Differential Geometry. Vol I, Interscience Publisher, New York-London, 1963.

[3] Kolář, I., Michor, P. W., Slovák, J., Natural Operations in Differential Geometry, Springer-Verlag, Berlin, 1993.

[4] Kurek, J., Mikulski, W. M., On lifting of connections to Weil bundles, Ann. Polon. Math. 103 (3) (2012), 319-324.

[5] Kurek, J., Mikulski, W. M., On almost complex structures from classical linear connections, Ann. Univ. Mariae Curie-Skłodowska Sect. A, 71 (1) (2017), 55-60.

[6] Libermann, P., Sur les structures presque paracomplexes, C. R. Acad. Sci. Paris 234 (1952), 2517-2519.

[7] Libermann, P., Sur le probleme d'equivalence de certaines structures infinitesimales, Ann. Mat. Pura Appl. 36 (1954), 27-120.

\author{
Anna Bednarska \\ Institute of Mathematics \\ Maria Curie-Skłodowska University \\ pl. M. Curie-Skłodowskiej 1 \\ 20-031 Lublin \\ Poland \\ e-mail: bednarska@hektor.umcs . lublin.pl
}

Received April 25, 2018 